Copyright (C) 2007 IEEE. Reprinted from Proceedings of the IEEE/ICME International Conference on Complex Medical Engineering (2007 : Beijing, China):pp.869-875

This material is posted here with permission of the IEEE. Such permission of the IEEE does not in any way imply IEEE endorsement of any of the University of Adelaide's products or services. Internal or personal use of this material is permitted. However, permission to reprint/republish this material for advertising or promotional purposes or for creating new collective works for resale or redistribution must be obtained from the IEEE by writing to pubs-permissions@ieee.org.

By choosing to view this document, you agree to all provisions of the copyright laws protecting it. 


\title{
Motion Estimation of Vortical Blood Flow Within the Right Atrium in a Patient with Atrial Septal Defect
}

\author{
Kelvin K.L. Wong ${ }^{1,2}$, P. Molaee ${ }^{2}$, P. Kuklik ${ }^{2}$, Richard M. Kelso ${ }^{3}$, S.G Worthley², P. Sanders ${ }^{2}$, \\ J. Mazumdar ${ }^{1}$ and D. Abbott ${ }^{1}$, Fellow, IEEE \\ ${ }^{1}$ Centre for Biomedical Engineering and School of Electrical \& Electronic Engineering, \\ The University of Adelaide, SA 5005, Australia. \\ ${ }^{2}$ Cardiovascular Research Centre and School of Medicine, \\ The University of Adelaide, SA 5005, Australia. \\ ${ }^{3}$ School of Mechanical Engineering \\ The University of Adelaide, SA 5005, Australia.
}

\begin{abstract}
Patients with an atrial septal defect (ASD) have a left to right shunt with associated complications. Currently, various imaging modalities, including echocardiography and invasive cardiac catheterization, are utilized in the management of these patients. Cardiac magnetic resonance (CMR) imaging provides a novel and non-invasive approach for imaging patients with ASDs. A study of vortices generated within the right atrium (RA) during the diastolic phase of the cardiac cycle can provide useful information on the change in the magnitude of vorticity pre- and post-ASD closure. The motion estimation of blood applied to CMR is performed. In this study we present, a two dimensional (2D) visualization of in-vivo right atrial flow. This is constructed using flow velocities measured from the intensity shifts of turbulent blood flow regions in MRI. In particular, the flow vorticities can be quantified and measured, against controls and patients with ASD, to extend medical knowledge of septal defects and their haemodynamic effects.
\end{abstract}

\section{INTRODUCTION}

The investigation of vortical blood flow in cardiovascular structures and cardiac chambers sets a new direction for the exploration of knowledge in the hemodynamics of blood within the human heart. In the literature there is limited discussion of the significance of vortices in relation to cardiac function, nevertheless we know that vortices are energy preserving flow structures and are observed in normal cardiac chambers [1]. It is believed that this improves the efficiency of the heart [2]. Flow information can be used to examine the amount of energy wasted by a heart with structural abnormalities such as ASD or valvular disease. The study of vortices in the human heart has been extensively performed using phase-contrast data [3]. The quantification of intracardiac flow using a velocity field gives information on the vortical blood flow.

A unique definition of vorticity is provided by examining the magnitude of rotation of fluid about a specific examined point [4]. However, this is limited to $2 \mathrm{D}$ analysis, whereas it has been well documented that vortices are seldom limited to a plane, but rather a three-dimensional (3D) space is required for their examination [5]. However, presentation of resolved vector components of spiraling flows or skewed vortical flow in a 3D space is visually complicated and will make the analysis of vorticity difficult. We have shown in our study that visual examination of vortices in CMR images of the human RA using 2D slices of the scan is sufficient for vortex quantification, as we will be comparing magnitude and occupancy of a single vortex on a specific slice and phase of the cardiac cycle for a patient who has been scanned pre- and post-septal occlusion.

Application of optical flow on MRI is carried out and the velocity fields are used in describing vorticity in cardiac chambers. In this paper, we present the optical flow implementation on time dependent intense turbulent blood movement in a cardiac chamber. We have used the pyramidal Lucas Kanade optical flow scheme [6] to measure intensity flow from MRI and present a dense $2 \mathrm{D}$ velocity field. Averaging of velocity components within sampling frames can be performed to vary the coarseness of the velocity field. The overall pyramidal algorithm proceeds as follows: first, the optical flow is computed at the coarsest pyramid level. Then the result of that computation is propagated to the next level in a form of an initial guess for pixel displacement, and this process is carried on until the original resolution of the image is reached.

From the definitions of vorticity, shear and normal strains, which are extrapolated from fluid mechanical concepts in Particle Image Velocim etry (PIV) [4], we compute the flow characteristics of the velocity field. We examine the intensity of vorticity for blood flow in the RA before and after septal occlusion by using a histogram representation of the pixel count in the flow field with respect to the vorticity, shear and normal strain magnitudes.

Based on our preliminary studies, we have discovered that all flow analyses carried out are based on the left instead of 
the right atrium. Although the study of vortices in the RA may not be of great utility in terms of cardiac related clinical end-points, such as thrombosis and stroke risk, it is nevertheless important to have an understanding of how the vortex is diminished during atrial left to right shunting. This presents new knowledge on atrial flow for ASD patients.

Using standard intensity-based CMR images that are obtained from the MR-scanner during pre-processing, and integrating the optical flow scheme into our system, we are able to quantify vorticity in the human RA. Our methodology facilitates the study of vortical behavior and its growth over time and in real-time during the post-processing stage when the CMR images are examined.

\section{LITERATURE REVIEW}

We examine some of the common imaging modalities that are used for diagnosing ASD patients. Current available assessments quantifying blood flow have not been able to resolve the vortices that exist during flow. For example, methods based on ultrasound technology provide only flow rate quantification.

\section{A. Echocardiogram}

The echocardiogram is an ultrasound scan of the heart that may be used for assessing ASD patients $[7,8]$. Using standard ultrasound scans, two-dimensional slices of the heart can be imaged that can be used in flow visualization. In addition to creating scans of the cardiovascular system, the echocardiogram can also produce an assessment of the velocity of blood and cardiac tissue at any arbitrary point using pulsed or continuous wave Doppler ultrasound. This allows assessment of abnormal communications between the left and right side of the heart, such as any shunting of blood through the ASD.

Ultrasound is sensitive to blood flow, which makes it potentially applicable to flow visualization. However, this technique is currently used only as a qualitative and not quantitative modality and is liable to error depending on the plane of imaging. Contrast agents exist as a suspension of microscopic gas bubbles, which are used to enhance ultrasound performance. Ultrasound is, however, an inexpensive alternative to MRI, PET and CT modalities.

\section{B. Cardiac Magnetic Resonance Imaging}

The assessment of ASD can be performed on CMR imaging [7]. Examination using spin-echo cine-MRI which gives a good intensity contrast for the cardiac wall is able to reveal ASD. In addition, gradient-echo T1-weighted cineMRI of cardiac chambers allows visualization of blood movement, based on the shifting intensity in the image as a result of signal voids due to de-phasing of nuclear spins. It is interesting to observe that this signal void, within the local turbulent regions, is the result of rapid flowing blood. As these local turbulent regions move along with the global flow of the blood, we will be able to track the magnitude and direction of movement and quantify the flow structure.
However, there has been no method that performs flow quantification and as such, only a qualitative examination of the flow can be performed.

\section{Application of Motion Estimation on Tagged MRI}

The application of optical flow techniques to medical images offers the potential for velocity measurement to become a commonly accessible technology. The determination of flow velocities is based on the intensity shifts of the pixels using the optical flow algorithm. The assessment of flow is accurate provided that a good time resolution is present. However, the number of phases per cardiac cycle will be dependent on the capabilities and specifications of the particular medical imaging device used.

To the best of our knowledge, there has been no application of optical flow on cine-MRI scans of blood in cardiac chambers. The use of optical flow for image scans in conjunction with tagged MRI has been suggested previously, but only wall motion is tracked [9]. Note that optical flow analysis has been performed on tagged MR images before, but most of the analyses are on the movement of the cardiac walls $[10,11,12]$ and not on the blood flow itself.

\section{COMPUTATION}

\section{A. Active Contouring}

Movement of cardiac tissues is effectively excluded by performing contour segmentation on the cardiac walls. The cardiac wall is segmented by placement of a $2 \mathrm{D}$ contour that forms a computationally elastic wall within the cardiac chamber. Migration of contour nodes from their origins onto the intense wall region is executed based on an energy minimization algorithm $[10,11]$.

Active contouring computes a more accurate contour-line description iteratively by describing the contour as an energy function $E_{\text {contour }}$. It receives information from the preceding contour line and applies energy balancing based on the internal and external energies of this line denoted by $E_{i n t}$ and $E_{\text {ext }}$ respectively to redefine the contour representation. The best fitted contour is one that corresponds to the minimum of this energy:

$$
E_{\text {contour }}=\int E_{\text {int }}+\int E_{\text {ext }}
$$

The initial curve can be anywhere in the image, and interior contours are automatically detected. In the event of poor segmentation due to over-expansion of the elastic contour, the internal wall of the RA is manually traced. Because of the semi-automatic nature of RA segmentation, contour tracing is pre-processed.

\section{B. Optical Flow}

The optical flow algorithm is a class of motion estimation methods. It generates flow vectors that correspond to the apparent motion of brightness or intensity patterns in the image [13]. We denote pixel intensity by $I(x, y, t)$. Assuming spatiotemporal variation in intensity signal, we obtain 


$$
I(x, y, t)=I(x+\delta x, y+\delta y, t+\delta t) .
$$

Applying chain rule for differentiation, equation (2) becomes

$I(x+\delta x, y+\delta y, t+\delta t)=I(x, y, t)+\frac{\partial I}{\partial x} \delta x+\frac{\partial I}{\partial y} \delta y+\frac{\partial I}{\partial t} \delta t+\varepsilon(3)$

whereby $\varepsilon$ represents higher order terms. If the brightness of a particular point in the pattern is constant, we have

$$
\frac{\partial I}{\partial x} \delta x+\frac{\partial I}{\partial y} \delta y+\frac{\partial I}{\partial t} \delta t=0
$$

Differentiation with respect to $t$ yields

$$
\frac{\partial I}{\partial x} \frac{\delta x}{\delta t}+\frac{\partial I}{\partial y} \frac{\delta y}{\delta t}+\frac{\partial I}{\partial t}=0
$$

Definition of $v_{x}=\frac{\delta x}{\delta t}$ and $v_{y}=\frac{\delta y}{\delta t}$ yields

$$
\frac{\partial I}{\partial x} v_{x}+\frac{\partial I}{\partial y} v_{y}+\frac{\partial I}{\partial t}=0
$$

Therefore, the optical flow constraint equation can be rewritten as

$$
\left(I_{x}, I_{y}\right) \cdot\left(v_{x}, v_{y}\right)=-I_{t}
$$

The optical flow vector $\vec{v}$ has two components $v_{x}$ and $v_{y}$ describing the motion of a point feature in $x$ and $y$ direction with the spatial gradient of intensity is denoted by $\nabla I$. Therefore, the linearized version of the brightness constancy assumption yields the optical flow constraint given by

$$
\nabla I \cdot \vec{v}=-I_{t}
$$

In our optical flow scheme, the pyramidal Lucas Kanade optical flow method [6] which incorporates a multi-scale approach has been applied to support large blood motion and for improved accuracy. A top-down estimation of the flow by using an image pyramid is performed, with the apex representing the CMR image at a coarse scale. Computational results from this level are passed to the next and this process is carried on based on the flow estimated at the preceding scale until the original scale is reached.

\section{Velocity and Vorticity Flow Field}

A dense velocity field (velocity vector per pixel) is displayed and that vector averaging for sampling window resolution of 3 by 3 pixels is carried out. Scaling is carried out with respect to all the vectors present in the displayed phases of the cardiac cycle for ease of visualization and referencing. However, for analysis of dominant vortices, the flow values which appeared within each segmented vortex per slice have their magnitudes scaled independently. Positive values signify clockwise rotation (CW), whereas negative values represent counter-clockwise (CCW) motion of the blood. These may be represented by a color scale with maximum and minimum values represented by red and blue respectively (as observed in the figures showing characteristic flow in this paper).

\section{Flow Characteristics}

Statistical quantification of non-linear flow, or turbulence, for blood flow in the atria can be performed by examining the vorticity $(\Gamma)$, shear strain $(\Phi)$ and normal strain $(\Psi)$ of the blood. These measures of non-linear flow are computed from the velocity vectors in the motion field produced by the optical flow scheme [4]. Of course, other measures of nonlinear flow could also be calculated within the scope of the present technique. Based on the velocity contour, with its $x$ and $y$ components as $V_{x}(i, j)$ and $V_{y}(i, j)$ respectively, about a point of interest located at $(i, j), N$ represents the number of layers of the contour within the sampling frame and $\Delta x$ and $\Delta y$ represent the horizontal and vertical distances between neighboring velocities. The flow characteristics can be numerically determined:

The vorticity $\Gamma$ represents the rotation of blood in the RA that we are examining here,

$$
\Gamma=\sum_{n=1}^{N}\left\{\frac{V_{x}(i+n, j)-V_{x}(i-n, j)}{2 n \Delta y}-\frac{V_{y}(i, j+n)-V_{y}(i, j-n)}{2 n \Delta x}\right\} .
$$

The shear strain $\Phi$ represents the shear that the blood experiences,

$$
\Phi=\sum_{n=1}^{N}\left\{\frac{V_{x}(i+n, j)-V_{x}(i-n, j)}{2 n \Delta y}+\frac{V_{y}(i, j+n)+V_{y}(i, j-n)}{2 n \Delta x}\right\} .
$$

The normal strain $\Psi$ determines pressure experienced by the blood at local positions and this has some implications in strain of the bio-fluid. It is given by,

$$
\Psi=\sum_{n=1}^{N}\left\{\frac{-V_{x}(i+n, j)+V_{x}(i-n, j)}{2 n \Delta y}+\frac{-V_{y}(i, j+n)+V_{y}(i, j-n)}{2 n \Delta x}\right\} .
$$

\section{METHODOLOGY}

\section{A. Subjectfor Case Study}

A male subject, aged 18 years at the time of this experiment, volunteered for this study and approval was gained from the institutional ethics committee. The subject had an ASD and was assessed using CMR. Implantation of the septal occluder device in the patient was performed 79 days later and another scan was carried out 210 days after the ASD occlusion.

\section{B. MRI Scan Procedure}

For this research, CMR imaging is performed using a Siemens Sonata, 1.5 Tesla, model - syngo MR 2004A scanner with Numaris - 4, Series No: 21609 software. Steadystate free precession cine-MR imaging was performed using contiguous slices in short axis views through the ASD. All images were acquired with retrospective gating and 25 phases (from $T=0$ to 24 ) for each slice are obtained. Acquisition parameters include: $\mathrm{TR}=47.1 \mathrm{~ms}, \mathrm{TE}=1.6 \mathrm{~ms}, \mathrm{FOV}=298$ $\times 340 \mathrm{~mm}$ at matrix of $134 \times 256$ pixels for pre-septal occlusion scans and $\mathrm{TR}=40.3 \mathrm{~ms}, \mathrm{TE}=1.2 \mathrm{~ms}, \mathrm{FOV}=260$ 
$\times 320 \mathrm{~mm}$ at matrix of $156 \times 192$ pixels for post-septal occlusion scans. The images for pre-septal occlusion are reduced to sizes of $156 \times 192$ pixels to match those of postseptal occlusion, in order to maintain consistency during computation and analysis.

\section{Clinical Diagnosis}

We select CMR image slices of the patient that corresponds to diastolic phases of the heart. We observed the vortices that are generated pre- and post-septal occlusion at the selected phases. The RA was enlarged due to a left to right shunt, and the changes in the signal intensity of blood using MRI are more easily detected than in the other chambers. A region of interest with width of 120 pixels and height of 150 pixels is truncated from the selected slice showing the sectioning of the right and left atria, where the ASD is most apparent, is to be displayed for qualitative examination.

\section{Data Analysis}

Based on a velocity field that is measured, a sampling frame of 21 by 21 windows, for the vorticity and strain calculations, is used. This corresponds to 10 layers of neighboring contours. Averaging of vector components within a 3 by 3 pixel window is carried out.

Statistical quantification of turbulence, for blood flow in the RA, is performed by examining the vorticity, shear strain, and normal strain of the blood. The measures of non-linear flow are computed from velocity vectors in the motion field produced by the optical flow scheme.

The size of a dilated RA due to a left to right shunt is different from that for a closed ASD. Even if there has been no dilation, it is still hard to maintain equal area size of the RA for every slice, due to the impossibility of a perfectly controlled comparison for scans, which are taking place on two occasions before and after a pathology that has been surgically treated. Normalization is a means of standardizing the total count of pixels within a segmented region of interest (the examined RA) and this is equal to sum of all bin counts of the histogram. Normalization of the histograms is performed by setting the sum of all counts in the bins to 3500 . The total counts correspond to the number of pixels representing the RA per slice. The number of bins is set at 35 .

\section{E. Characterization of Dominant Vortex}

The occupied area of each of the dominant vortices within the RA is visually determined. After contouring the vortex, the magnitude of this vortex can be measured and characterized. We provide a display of vortex occupancy for a single slice at multiple phases and at multiple slices at a single phase for both pre- and post-septal occlusion scans. Vorticity averaging for sampling window resolution of 3 by 3 pixels is carried out.

In our analysis, we have provided a series of vortices for a number of slices and phases. We then isolate and quantify the dominant vortex at different slices and phases for characterization and analysis. Scaling for the segmented vortex is carried out independent of every slice and phase of a cardiac cycle, and should not be referenced visually against other images from different slices or cardiac phases.

\section{RESULTS}

In this section, we discuss the flow patterns of blood in RA by analyzing the magnitude and dimension of the vortices in the image. It is proposed that the vortices in a patient with an ASD will be enlarged after septal occlusion. This can be visually observed in Appendices A and B which provides the intensity-based CMR images of scans (pre- and post-septal occlusion) for slice 2 from $T=8$ to 13 . An indication of the intensity of vorticity can be provided by generating histograms depicting the counts of pixels on the vertical axis with their respective values of vorticity, normal strain, and shear strain in the horizontal scale.

We select four MR image slices from the contiguous scans in short axis views through the ASD as illustrated in Fig. 1. Analyses of atria flow are carried out during the diastolic phases of the heart. This corresponds to phases that occur for maximum left to right shunting of blood during atria systole.

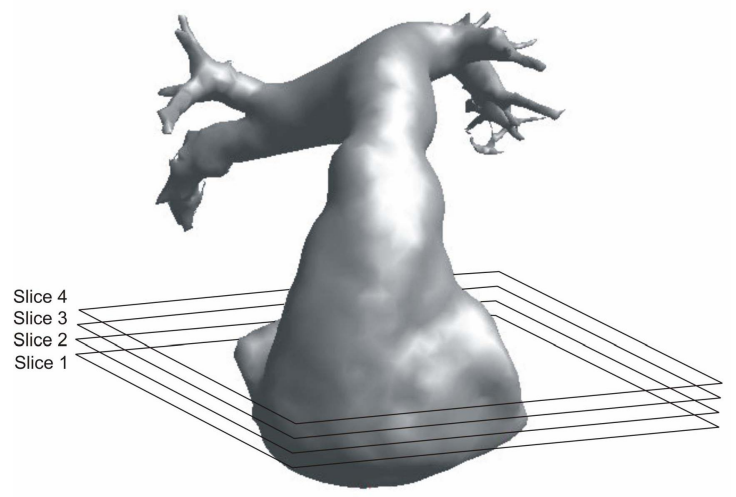

Fig. 1. CMR scans of the cardiac chambers were performed in the short axis view at space intervals of $6 \mathrm{~mm}$ starting from the apex of the heart. A 3D reconstruction of the RA from these scans has been performed. The selected MR images that correspond to the sectioning of left and right atria are labeled as slice numbers 1 to 4 are used for examination and flow analyses.

The observation of vortices and statistical analyses are performed for the RA and displayed on the left and the right respectively in all the figures depicting the flow results. The global estimation of flow velocity vector fields over the whole image provides useful information on the presence of vortices within the RA that can be used to assess the vorticity. The magnitude of vortical flow can be determined from the mean of vorticity histogram, $\bar{\Gamma}$, and the shear strain $\bar{\Phi}$ and normal strain, $\bar{\Psi}$ that are associated with it. The results using CMR images at slice 2 for pre- and post-septal occlusion are shown in Figs. 2 and 3 respectively. The examination of dominant vortices by excluding all other vortices is illustrated in Figs. 4 and 5 for pre- and post-septal occlusion respectively. Fig. 6 displays the visualization of dominant vortex for all the four slices at a specific time phase, $T=11$. The area occupied by the dominant vortex is determined by tracing its contour along its boundary of zero vorticity or edge of the cardiac chamber. 

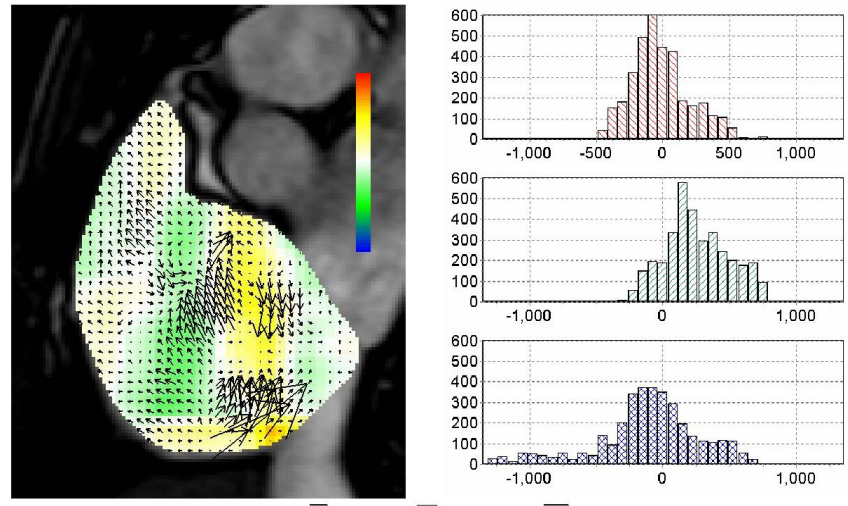

(a) $T=9 \quad \bar{\Gamma}=-9.99$
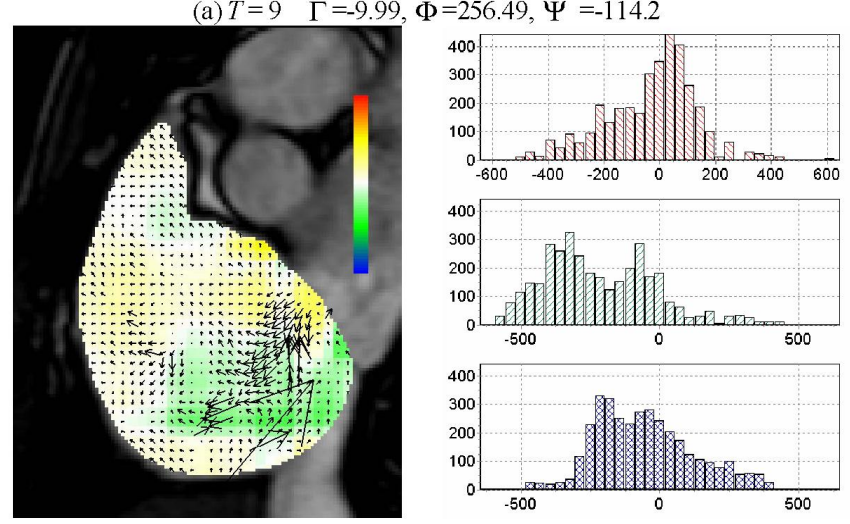

(b) $T=10 \quad \bar{\Gamma}=-30.21, \bar{\Phi}=-211.04, \bar{\Psi}=-53.67$
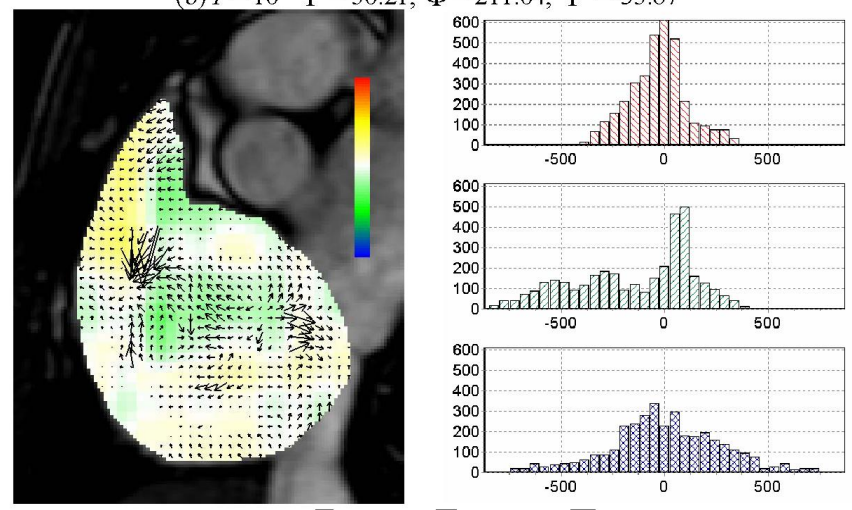

(c) $T=11 \quad \bar{\Gamma}=-32.94, \bar{\Phi}=-141.69, \bar{\Psi}=2.12$
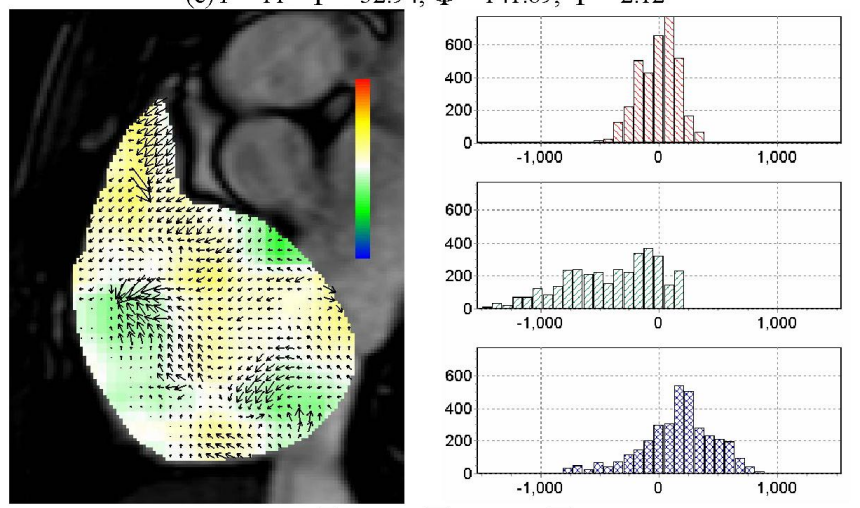

(d) $T=12 \quad \bar{\Gamma}=-6.88, \bar{\Phi}=-404.9, \bar{\Psi}=157.27$

Fig. 2. Vorticity visualization from CMR scans of segmented RA, pre-septal occluder insertion at 4 phases $(T=9,10,11,12)$ of cardiac cycle. The flow characteristic is shown for vorticity, normal strain, and shear strain starting from top to bottom. Note that scale for vorticity ranges from -1360 (CCW) to $+1288(\mathrm{CW})$. Vector averaging (resolution of 3 by 3 pixels) is carried out.
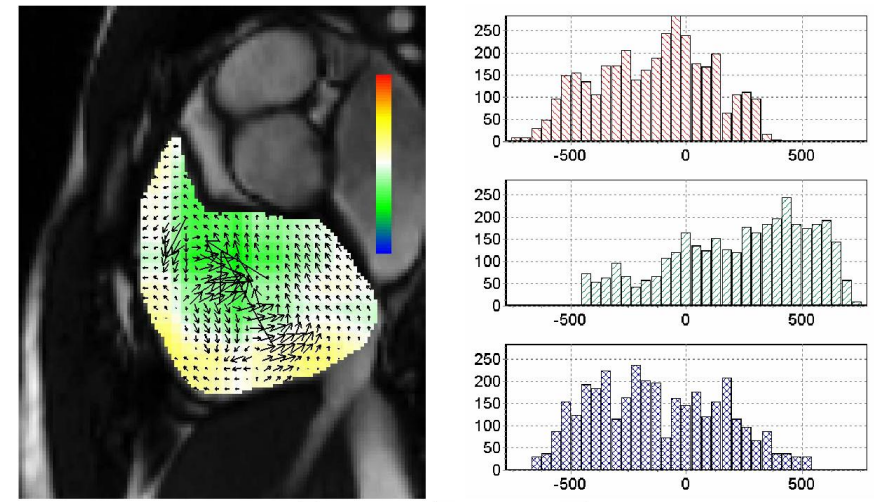

(a) $T=9 \quad \bar{\Gamma}=-142.98, \bar{\Phi}=231.09, \bar{\Psi}=-125.25$
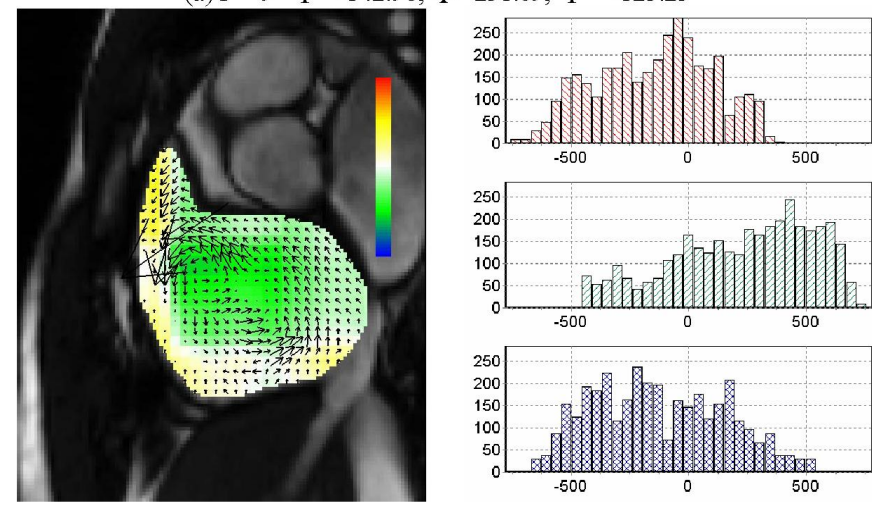

(b) $T=10 \quad \bar{\Gamma}=-241.39, \bar{\Phi}=-8.58, \bar{\Psi}=25.82$
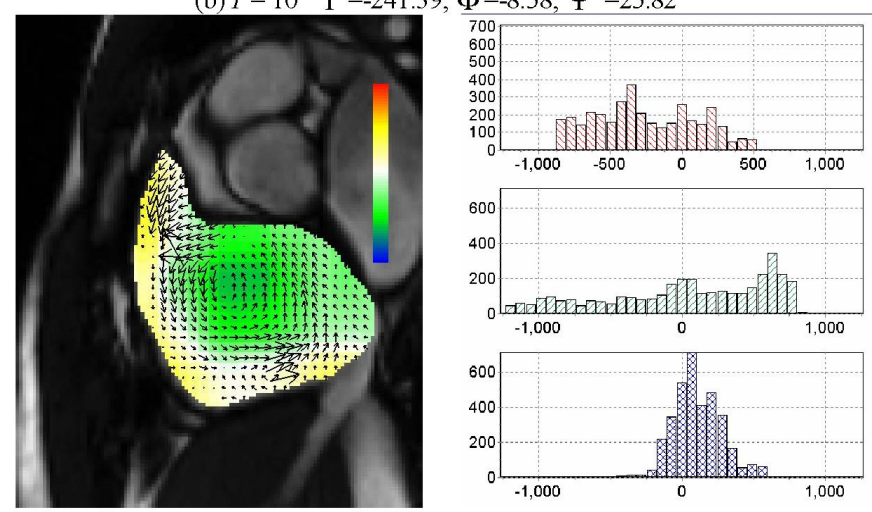

(c) $T=11 \quad \bar{\Gamma}=-253.54, \bar{\Phi}=40.92, \bar{\Psi}=108.74$
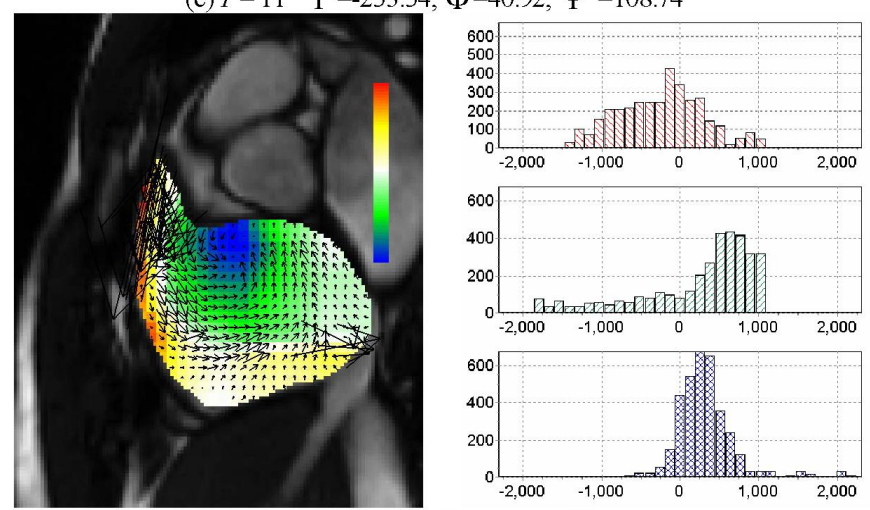

(d) $T=12 \quad \bar{\Gamma}=-234.93, \bar{\Phi}=236.61, \bar{\Psi}=329.44$

Fig. 3. Vorticity visualization from CMR scans of segmented RA, post-septal occluder insertion at 4 phases $(T=9,10,11,12)$ of cardiac cycle. The flow characteristic is shown for vorticity, normal strain, and shear strain starting from top to bottom. Note that scale for vorticity ranges from -1360 (CCW) to +1288 (CW). Vector averaging (resolution of 3 by 3 pixels) is carried out. 

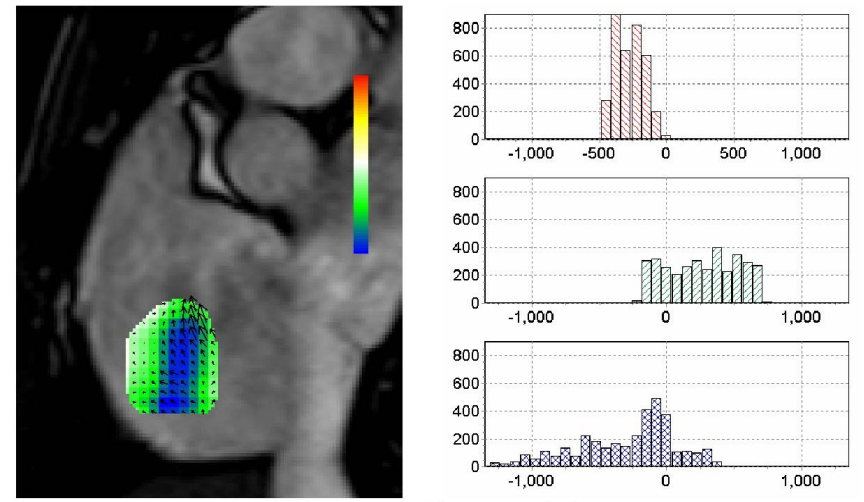

(a) $T=9 \quad \bar{\Gamma}=-274.06, \bar{\Phi}=266.65, \bar{\Psi}=-296.24$
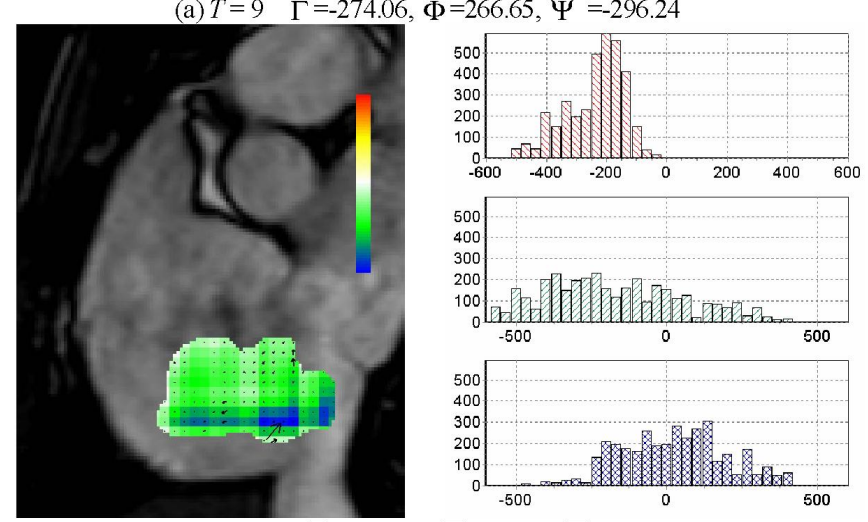

(b) $T=10 \quad \bar{\Gamma}=-218.63, \bar{\Phi}=-224.5, \bar{\Psi}=61.56$
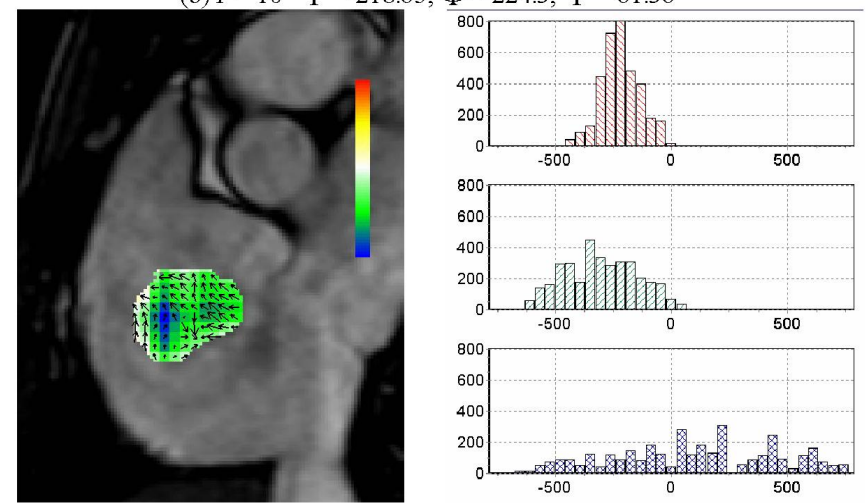

(c) $T=11 \quad \bar{\Gamma}=-217.25, \bar{\Phi}=-297.67, \bar{\Psi}=94.99$
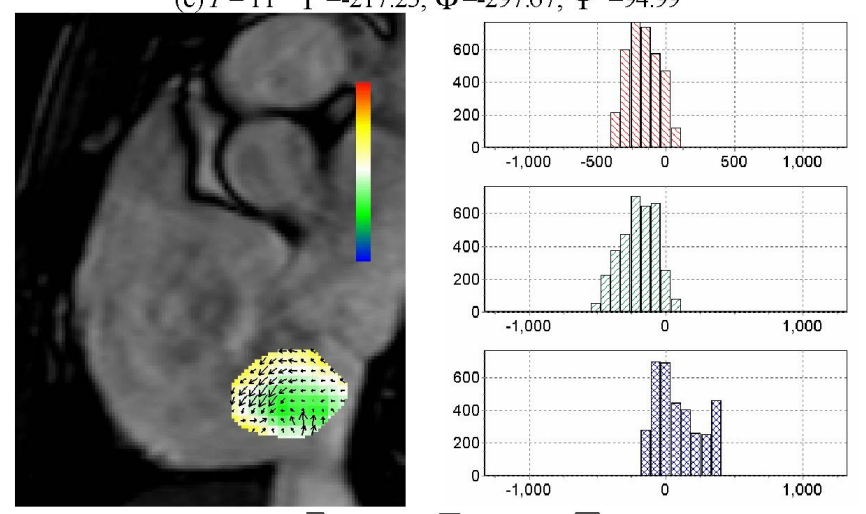

(d) $T=12 \quad \bar{\Gamma}=-141.03, \bar{\Phi}=-255.06, \bar{\Psi}=37.17$

Fig. 4. Vorticity visualization from CMR scans of dominant vortex within RA, pre-septal occluder insertion at 4 phases $(T=9,10,11,12)$ of the cardiac cycle. The flow characteristic post-septal occlusion is shown for vorticity, normal strain and shear strain starting from the top to the bottom. Note that scale for vorticity for each image is independent of the other slices and phases. Vector averaging (resolution of 3 by 3 pixels) is carried out.
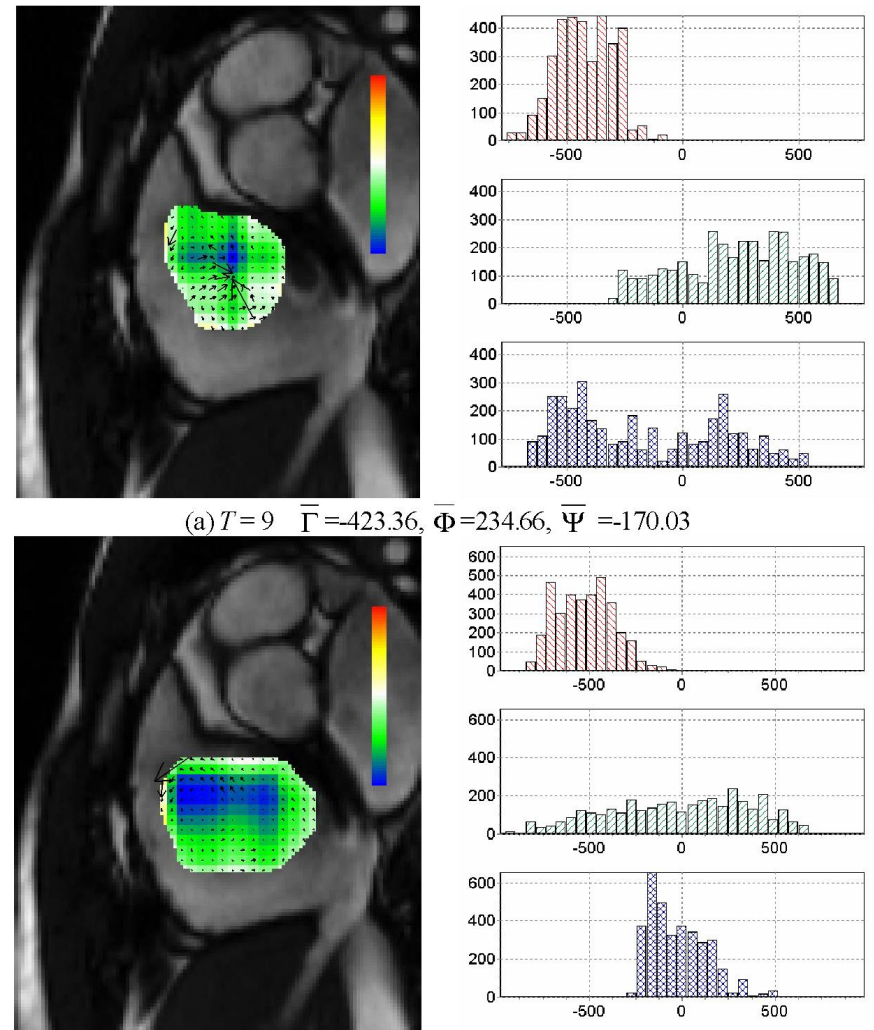

(b) $T=10 \quad \bar{\Gamma}=-522.4, \bar{\Phi}=-16.8, \bar{\Psi}=-19.54$
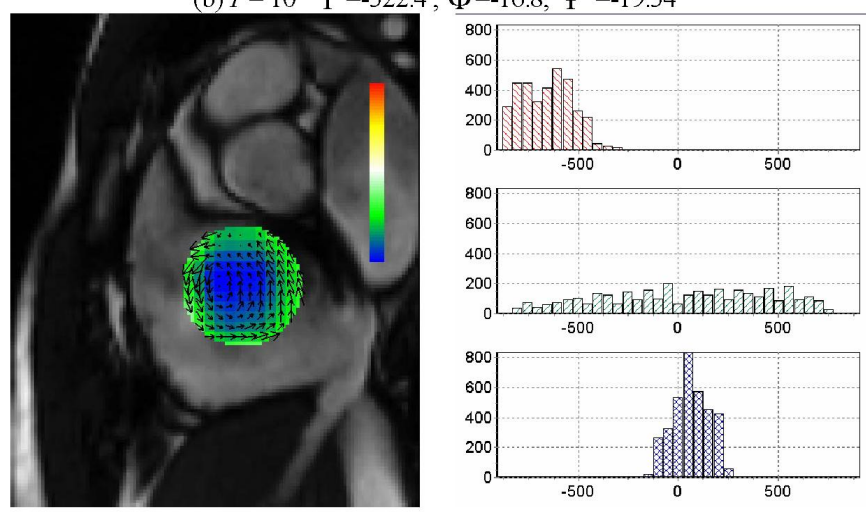

(c) $T=11 \quad \bar{\Gamma}=-656.8, \bar{\Phi}=35.16, \bar{\Psi}=63.88$
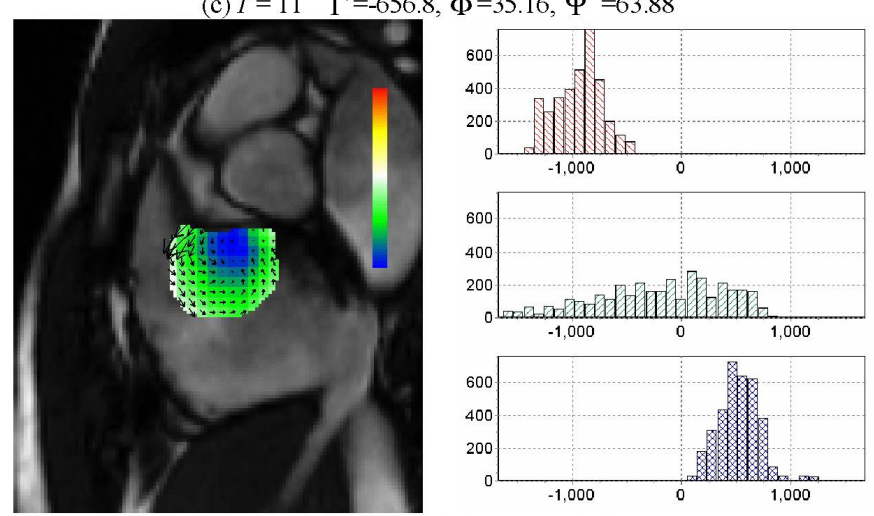

(d) $T=12 \quad \bar{\Gamma}=-945.78, \bar{\Phi}=-192.25, \bar{\Psi}=529.43$

Fig. 5. Vorticity visualization from CMR scans of dominant vortex within RA, post-septal occluder insertion at 4 phases $(T=9,10,11,12)$ of the cardiac cycle. The flow characteristic post-septal occlusion is shown for vorticity, normal strain, and shear strain starting from the top to the bottom. Note that scale for vorticity for each image is independent of the other slices and phases. Vector averaging (resolution of 3 by 3 pixels) is carried out. 

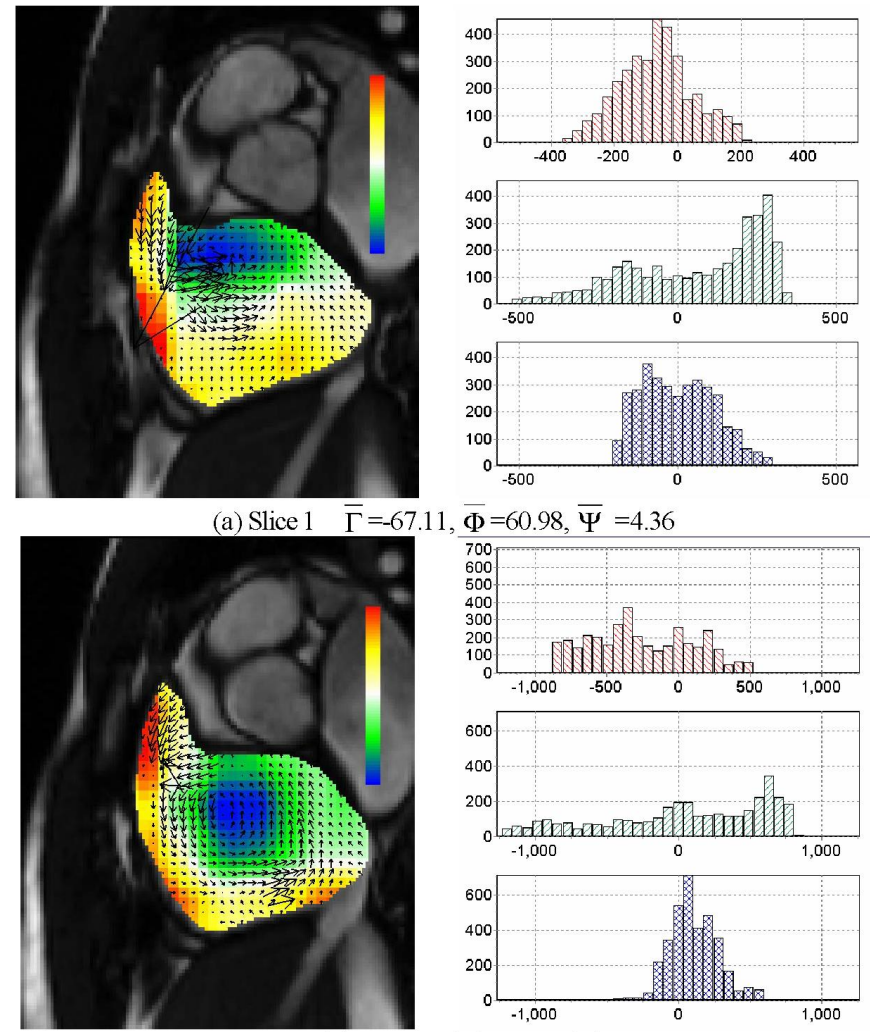

(b) Slice $2 \quad \bar{\Gamma}=-253.54$
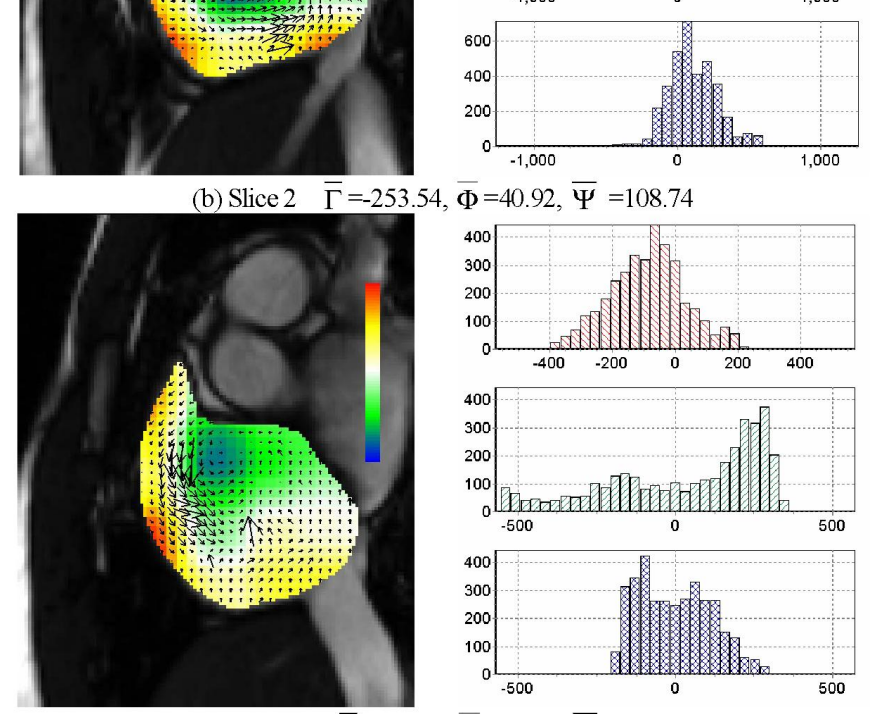

$\bar{\Phi}=40.92, \bar{\Psi}=108.74$
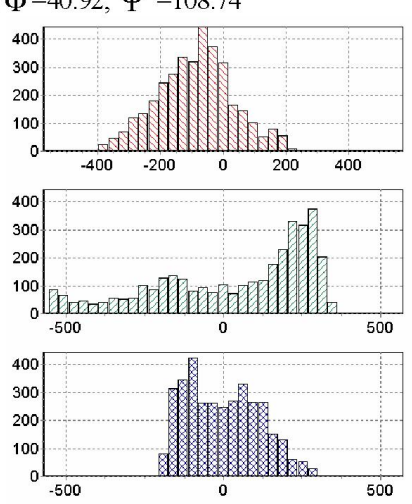

(c) Slice $3 \quad \bar{\Gamma}=-86.97, \bar{\Phi}=33.91, \bar{\Psi}=-0.47$
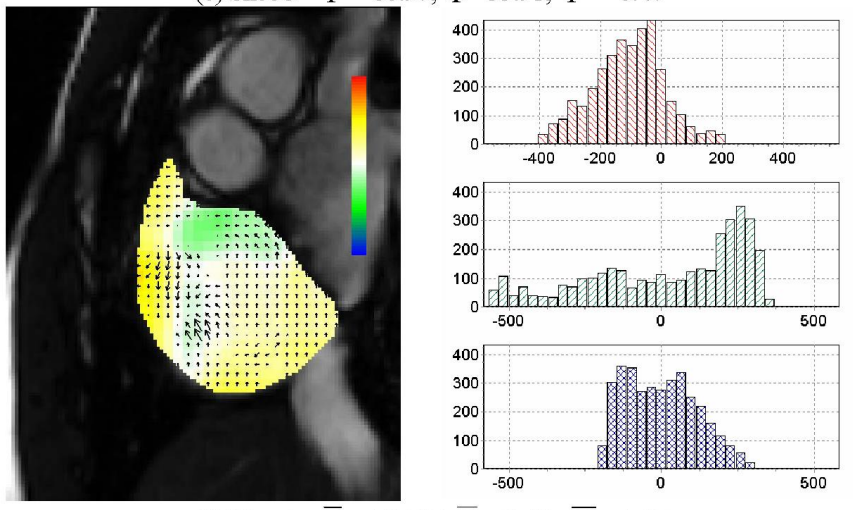

(d) Slice $4 \quad \bar{\Gamma}=-107.05, \bar{\Phi}=21.03, \bar{\Psi}=1.07$

Fig. 6. Vorticity visualization from CMR scans of vortices within RA at multiple slices, pre- and post-septal occluder insertion at one phase $(T=11)$ of the cardiac cycle. Note that slice number starts from the apex of the heart working upwards. Scale for vorticity ranges from $-875(\mathrm{CCW})$ to $+519(\mathrm{CW})$ Vector averaging (resolution of 3 by 3 pixels) is carried out.

\section{DISCUSSION}

The intensity of vorticity gives a good estimation of the swirl structure in the RA. This is also correlated with the shear and normal strain that is experienced by the blood. An energy preserving vortex will have a high value of $\bar{\Gamma}$, but low values of $\bar{\Phi}$ and $\bar{\Psi}$, as most of the energy is channeled towards the rotation and not in the transverse direction to generate strains within the fluid. Note that our definition of vorticity, shear strain and normal strain magnitudes is based on absolute values of $\bar{\Gamma}, \bar{\Phi}$ and $\bar{\Psi}$. Therefore, we ignore the negative signs in our analysis. We shall discuss this in detail.

In Fig. 2, flow is characterized by low magnitudes of $\bar{\Gamma}$ in range of 6.88 to 32.94 and relatively high magnitudes of $\bar{\Phi}$ and $\bar{\Psi}$ from ranges of 141.69 to 404.9 and 2.12 to 157.27 respectively. This is different to flow characteristics of Fig. 3, whereby $\bar{\Gamma}$ has a much higher range of 142.98 to 253.54 and a relatively lower magnitude of $\bar{\Phi}$ from 8.58 to $236.93 . \bar{\Psi}$ has a much greater variance and ranges from 25.82 to 329.44 .

Isolation of the dominant vortex in Fig. 4 does not raise the magnitude of vorticity with respect to its strains significantly. $\bar{\Gamma}, \bar{\Phi}$ and $\bar{\Psi}$ have ranges of 141.03 to $274.06,224.5$ to 297.67 and 37.17 to 296.24 respectively. A good reference for high vorticity can be assessed in slice 2 at $T=11$ shown in Fig. 5 (b) after septal occlusion. Its high magnitude of $\bar{\Gamma}=656.8$ for vorticity relative to the strain magnitudes of $\bar{\Phi}=35.16$ and $\bar{\Psi}$ $=63.88$ is significant when compared to the magnitude of blood rotation before septal occlusion which has a lower magnitude of $\bar{\Gamma}=217.25$ and relative to higher magnitudes of $\bar{\Phi}=297.67, \bar{\Psi}=94.99$.

In Figs. 3, 5 and 6, we observe that vorticity is increased in the RA for the same patient and at the same cardiac phase after septal occluder insertion. We also note the enlargement of the RA before septal occlusion in Figs. 2 and 4. We observe that there is an increase in vorticity of flow and a more defined vortex within the human RA despite a decrease in chamber size after septal occlusion. The corresponding strains generated by the vortical flow have low magnitudes.

\section{RESEARCHNOVELTY}

As an alternative to established medical imaging-based diagnostic and prognostic techniques discussed in literature, we have applied the optical flow scheme to MR images and performed flow quantification to analyze, in particular, the blood movement in the RA based on the measured flow field. As such, this technique offers potential for non-invasive flow visualization in cardiac structures before and after a septal occluder device insertion for ASD patients.

There are currently no techniques used in current medical imaging modalities for the assessment of blood stress and strain to the best of our knowledge. We have developed this optical-based measurement technique specifically for analyzing flow characteristics within the human heart in pathological conditions. 


\section{CONCLUSION}

We have examined vorticity during the diastolic phase for a heart with an ASD (pre- and post-septal closure). Based on the calculations of vorticity, shear strain, and normal strain, it is possible to measure the intensity of vorticity that exists in the RA. This increases due to septal closure and therefore allows assessment of the success of ASD closure. The technique may also be used to assess growth of cardiac tissue into the mesh of the occluder by examining blood leakage through the ASD. As quantitative as well as qualitative data is provided for flow, it serves potentially as a prognostic system for medical experts to assess surgical success.

The display of information using histograms of vorticity, shear, and normal strains provides an overview of the swirl and stress patterns present within the blood flow. We have also discovered that all the dominant vortices within the RA are in the counter-clockwise direction. This is consistent with our investigation of all other ASD patients, although we have not published their results here. Nevertheless, at the preliminary stage, the study of vortex formation and characterization of vorticity using the method highlighted in this paper still requires more investigation and verification.

We note that the occlusion is not a perfect one, as we are able to observe some blood leakage from the left to right atria, but it has nevertheless enabled us to assess the increase in vorticity against that of a heart with an ASD.

Although we have visually examined the rotation of blood in the selected CMR image slices and cardiac phases and observed that the velocity field, measured by the optical flow scheme, is consistent with the movement of blood based on its varying intensity, there has nevertheless, been no groundtruth data to verify its accuracy. For future verification, one good suggestion would be the comparison of velocity flow fields scanned using phase contrast MRI with those produced by the proposed system.

\section{APPENDIX}

\section{A. CMR Images of ASD patient (pre-septal occlusion)}
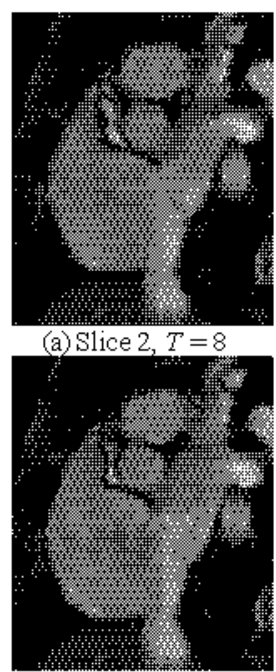

(d) Slice 2, $T=11$
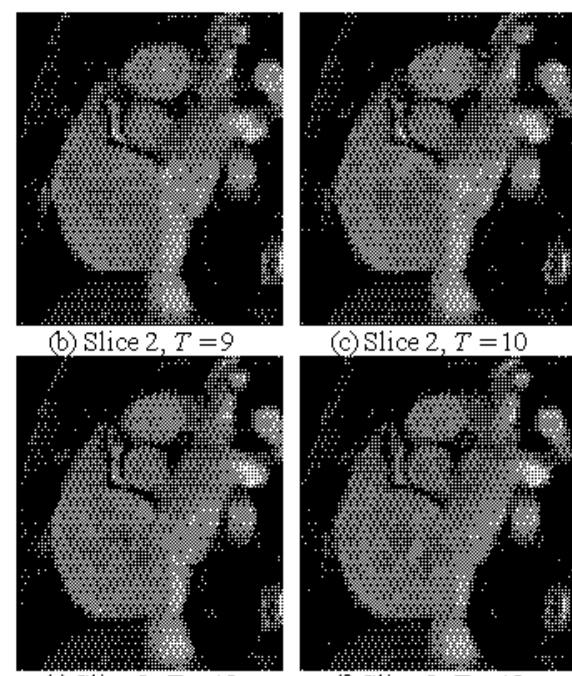

(e) Slice $2, T=12$

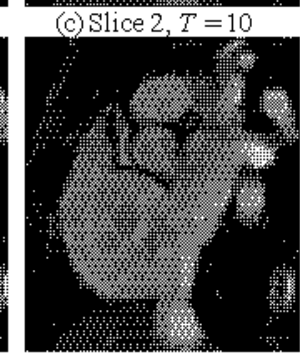

(f) Slice $2, T=13$

\section{B. CMR Images of ASD patient (post-septal occlusion)}
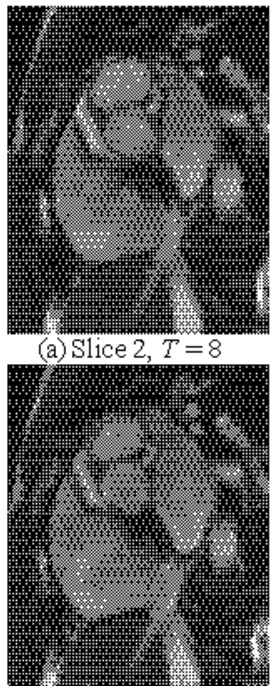

(d) Slice $2, T=11$
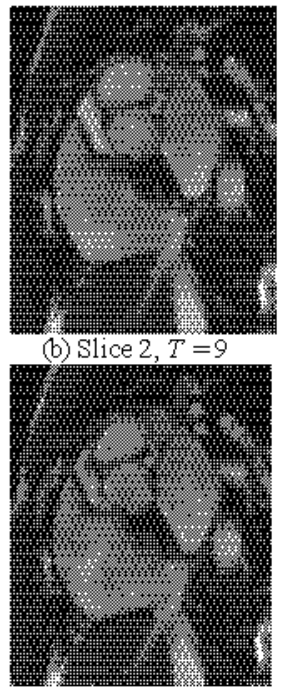

(e) Slice $2, T=12$

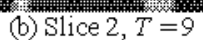

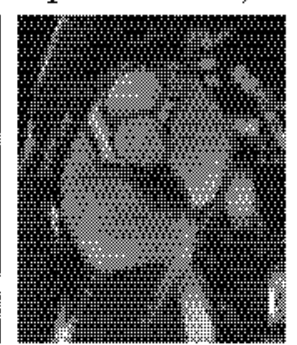
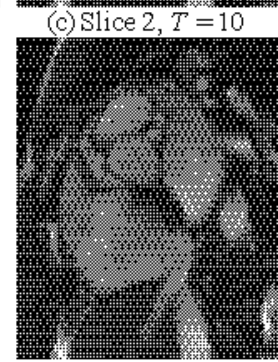

(f) Slice $2, T=13$

\section{ACKNOWLEDGMENT}

We thank the medical team at Royal Adelaide Hospital and Wakefield Hospital for scanning and supply of CMR images.

\section{REFERENCES}

[1] A Fyrenius, L Wigström, T Ebbers, M Karlsson, J Engvall and A F Bolger, "Three dimensional flow in the human left atrium", Heart, vol. 86 , pp. $448-455,2001$

[2] O. Pierrakos, P. P. Vlachos, "The effect of vortex formation on left ventricular filling and mitral valve efficiency", Journal of Biomechanical Engineering-Transactions of the ASME, vol. 128 (4), pp. 527-539, Aug 2006

[3] G.Z. Yang, R.H. Mohiaddin, P. J. Kilner, and D. N. Firmin, "Vortical Flow Feature Recognition: A Topological Study of In Vivo Flow Patterns Using MR Velocity Mapping", Journal of Computer Assisted Tomography, vol. 22 , pp. $577-586,1998$.

[4] M. Raffel, C. Willert, J. Kompenhans, Particle Image Velocimetry, Springer, Germany, 1998

[5] E. Brandt, T. Ebbers, L. Wigström, J. Engvall and M. Karlsson, "Automatic Detection of Vortical Flow Patterns from Threedimensional Phase Contrast MRI", Proc. Intl. Soc. Mag. Reson. Med, vol. 9 , pp. $1838,2001$.

[6] J.Y. Bouguet, "Pyramidal implementation of the lucas kanade feature tracker", OpenCV documentation, Microprocessor Research Labs, Intel Corp., 2000

[7] G. Webb and MA Gatzoulis, "Atrial septal defects in the adult - Recent progress and overview", Circulation, vol. 114 (15), pp. 1645-1653, Oct 2006

[8] C. J. McMahon, T. F. Feltes, J. K. Fraley, J. T. Bricker, R. G. Grifka, T A. Tortoriello, R. Blake and L. I. Bezold, "Natural history of growth of secundum atrial septal defects and implications for transcatheter closure", Heart, vol. 87 (3), pp. 256-259, Mar 2002.

[9] K. W. Moser, J. G. Georgiadis, and R. O. Buckius, "On the use of optical flow methods with spin-tagging magnetic resonance imaging," Ann. Biomed. Eng., vol. 29, p. 9, 2001

[10] L. D. Cohen, "On active contour models and balloons," Computer Vision, Graphics, and Image Processing, vol. 53(2), pp. 211-218, 1991.

[11] S. S. Jasjit, "Computer vision, pattern recognition and image processing in left ventricle segmentation: The last 50 years," Pattern Anal. Appl., vol. 3(3), pp. 209-242, 2000

[12] M. Sühling, M Arigovindan, C. Jansen, and et al., "Myocardial motion analysis from B-mode echocardiograms," IEEE Trans. on Img. Proc. vol. 14(4), pp. 525-536, 2005

[13] B. K. P. Horn and B. G. Schunck, "Determing optical-flow," Artificial Intelligence, vol. 17 (1-3), pp. 185-203, 1981 